\title{
Acute shock caused by Clonorchis sinensis infection: a case report
}

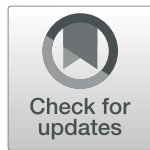

Nan Wang ${ }^{1 \dagger}$, Bin Tang ${ }^{1 \dagger}$, Yuhua Hao ${ }^{2}$, Xue Bai ${ }^{1}$, Xuelin Wang ${ }^{1}$, Yuxiang $\mathrm{Li}^{3}$, Yong Yang ${ }^{1}$, Shicun Li ${ }^{1}$, Shuo Hao ${ }^{1}$, Xinyu Wang ${ }^{1}$, Mingyuan Liu $^{1,4^{*}}$ and Xiaolei Liu ${ }^{1 *}$ (D)

\begin{abstract}
Background: Clonorchiasis, caused by Clonorchis sinensis (C. sinensis) infection, is a serious food-borne zoonotic disease that is often asymptomatic or shows only mild symptoms, which leads to delayed treatment and chronic clonorchiasis and results in various complications, such as cholelithiasis, cholangitis, cholecystitis and cholangiocarcinoma. However, acute shock caused by $C$. sinensis infection has not been reported. Here, for the first time, we describe a fatal case of acute shock caused by $C$. sinensis infection.

Case presentation: A patient with a history of eating raw or undercooked freshwater fish was hospitalized with acute shock caused by severe abdominal pain. Physical examination suggested acute abdomen with severe abdominal pain and rigidity. Computed tomography (CT) detection indicated acute cholecystitis and cholelithiasis. After cholecystectomy, several liver flukes were found in the drainage tube. Furthermore, morphological analysis and polymerase chain reaction (PCR) identified the pathogen as C. sinensis. The liver gradually restored normal function after anthelmintic therapy with praziquantel.

Conclusions: In this fatal case, C. sinensis infection was the cause of acute shock, which is rarely found in the clinic environment. This report aims to increase awareness of the hazards and complications related to clonorchiasis. The PCR diagnosis method used in this report might be helpful in reducing the misdiagnosis of clonorchiasis and unnecessary cholecystectomy.
\end{abstract}

Keywords: Clonorchiasis, Cholecystitis, Acute shock, PCR

\section{Background}

Clonorchiasis is a serious food-borne parasitic disease caused by $C$. sinensis infection, and humans become infected by $C$. sinensis after ingesting raw or semi-raw freshwater fish and shrimp that contain infective metacercariae [1]. In 2009, the International Agency for Research on Cancer classified C. sinensis as group I carcinogen [2]. Approximately 15 million people are infected with $C$. sinensis worldwide, especially in Southeast Asia, with 13 million people being infected in China alone [3, 4]. Clonorchiasis is often asymptomatic or shows only mild symptoms, such as indigestion, nausea, diarrhea, and abdominal discomfort [5]. The absence of typical symptoms may delay the

\footnotetext{
*Correspondence: liumy36@163.com; liuxlei@163.com

${ }^{+}$Nan Wang and Bin Tang contributed equally to this work.

${ }^{1}$ Key Laboratory of Zoonosis Research, Ministry of Education, Institute of

Zoonosis/College of Veterinary Medicine, Jilin University, Changchun 130000,

China

Full list of author information is available at the end of the article
}

diagnosis and treatment of the disease and may eventually lead to chronic infection. Currently, the gold standard for diagnosing $C$. sinensis infection is detecting eggs in stool [6]. However, the likelihood of misdiagnosing C. sinensis is high due to the low sensitivity of the detection of eggs in stool, and misdiagnosis could lead to chronic infection. Chronic infection results in biliary obstruction, cholelithiasis, cholangitis and cholecystitis, which are risk factors for cholangiocarcinoma (CCA) $[7,8]$. In the coming decades, nearly 5000 cases of CCA attributed to $C$. sinensis infection could occur in East Asia annually [9]. The obstruction of the biliary tract may lead to a bile and pancreatic juice countercurrent, which results in cholecystitis and pancreatitis $[10,11]$. However, acute shock caused by C. sinensis infection has not been reported. In this report, we present a fatal case of acute shock caused by $C$. sinensis infection. The PCR diagnosis method used in this report might be helpful for the diagnosis of clonorchiasis.

(c) The Author(s). 2019 Open Access This article is distributed under the terms of the Creative Commons Attribution 4.0 International License (http://creativecommons.org/licenses/by/4.0/), which permits unrestricted use, distribution, and 


\section{Case presentation}

A 52-year-old man who lived in Songyuan city, Jilin province, China, near the Songhua River and it is an endemic area of clonorchiasis. The man had an approximately twenty years history of eating raw freshwater fish during the annual summer fishing season but no history of other diseases, and he was hospitalized with acute shock caused by severe abdominal pain. After rescue, physical examination suggested the signs and symptoms of the patient included acute abdomen with severe abdominal pain in the right upper abdomen and rigidity, a drop in body temperature $\left(35.2^{\circ} \mathrm{C}\right)$, hyperhidrosis, vomiting, cyanosis of the mouth, increased respiratory rate (30-35 breaths per minute), increased heart rate (96-110 beats per minute), and decreased blood pressure $(60-90 / 40-60 \mathrm{mmHg})$. Computed tomography (CT) revealed cholecystitis, and stone-like substances were observed in the gallbladder (Fig. 1), with no obvious symptoms in other abdominal organs. Magnetic resonance cholangiopancreatography (MRCP) revealed the same changes as seen in the CT results. Laboratory tests showed a leukocyte count of $7.03 \times 10^{9} / \mathrm{L}$ (normal range, $\left.3.5-9.5 \times 10^{9} / \mathrm{L}\right)$ with an eosinophil percentage of 15.1 (normal range, $0.4-8.0 \%$ ) and total bilirubin of $230.3 \mu \mathrm{mol} / \mathrm{L}$ (normal range, $6.8-30.0 \mu \mathrm{mol} / \mathrm{L}$ ) with direct bilirubin of $116.9 \mu \mathrm{mol} / \mathrm{L}$ (normal range,0.0$8.6 \mu \mathrm{mol} / \mathrm{L}$ ). Based on the clinical results and CT findings, we diagnosed the patient with a case of acute cholecystitis and cholelithiasis. Exploratory laparotomy and cholecystectomy were performed after admission. However, no stone-like substances were found. Instead, six liver flukes and parasite eggs were found in the bile. We found the morphology of the liver flukes to be consistent with $C$. sinensis, with an anterior oral sucker and a centrally located ventral sucker, dorso-ventrally flattened, slightly narrow front, blunt round rear, similar to

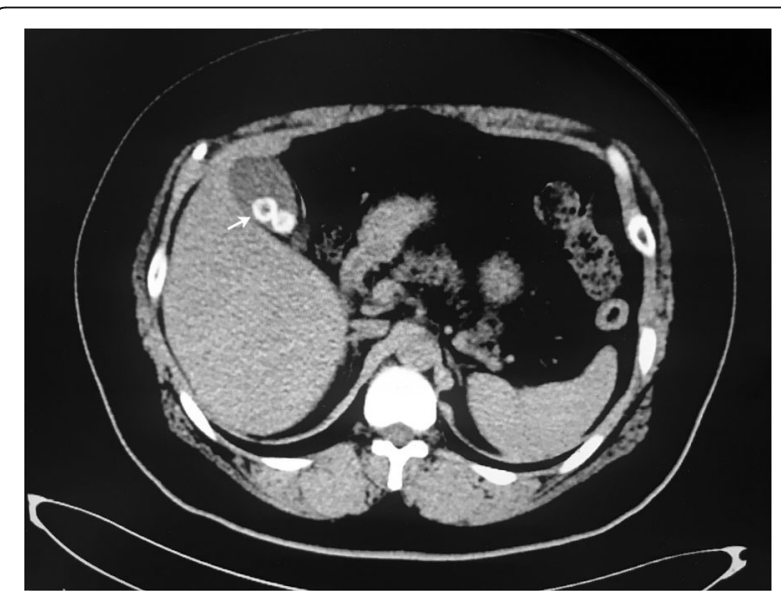

Fig. 1 CT image showing stone-like substance in the gallbladder (white arrow) a sunflower seed (Fig. 2). Eggs were similar to sesame seeds, light brown, with a thickening in the egg shell covering one side to form the shoulder (Fig. 2). Further, PCR was performed to identify the genomic DNA of the worms and eggs. Primers were designed based on the internal transcribed spacer 2 (ITS2) (GenBank No. JQ048577.1) of C. sinensis using Primer 5.0 software, and the fragment size was 860 bp (Fig. 3). Primer sequences are as follows: Primer F, TACCCAATATATATGATGTGC, and primer R, GAAAGTTAAGCACCGACCGGTGC. The sequencing results indicated that the DNA had 98\% homology with C. sinensis China Heilongjiang isolates (GenBank no. KF740424.1). Therefore, the patient was diagnosed with clonorchiasis. The results of two blood cultures before and after the operation were negative. After emergency surgery, other examinations, including urine routine, urine culture, lung CT, stool routine, and CRP and PCT levels, were performed, and no other potential infectious lesions were found. The electrocardiogram was normal and did not support shock caused by myocardial infarction or cerebral infarction. Other possible causes of shock in this patient were ruled out. In addition, the prognosis was good after treating the patient with praziquantel for 30 days; no eggs were found in the stool, and the liver gradually restored normal function. Therefore, we concluded that $C$. sinensis infection may have been the primary cause of shock in this case.

\section{Discussion and conclusions}

Here, we report a case of fatal shock caused by $C$. sinensis infection. C. sinensis seriously affects the health of people of East Asia, including those in China. National surveys on the status of serious human parasitic diseases in China demonstrated egg positive rates of 1.37-2.40\% from 1990 to 2003 in the epidemic area [12]. It is predicted that 12.49 million people in mainland China have been infected with C. sinensis. The highly prevalent areas of clonorchiasis are found in the northeastern provinces of China, such as Liaoning, Jilin, Heilongjiang [1]. A survey in select townships of Jilin province demonstrated that fecal samples from approximately $8.90 \%$ of 910 people tested contained C. sinensis eggs [1]. Our recent epidemiological surveys indicated that the prevalence of egg-positive individuals was $29.40 \%$ of 1745 people by the Kato-Katz (KK) method in three townships near the Songhua River of Songyuan city, Jilin province (data unpublished). However, there is a paucity of comprehensive epidemiological information for Jilin province. For the past decade, several cases of clonorchiasis have been reported in Jilin province [13, 14]. Even with the high prevalence of clonorchiasis, few cases are diagnosed in clinical examination. Due to the non-specific and atypical symptoms and the fact that adult worms survive in the human body for up to 26 years, the disease has always been neglected and often leads to clonorchiasis $[15,16]$. 


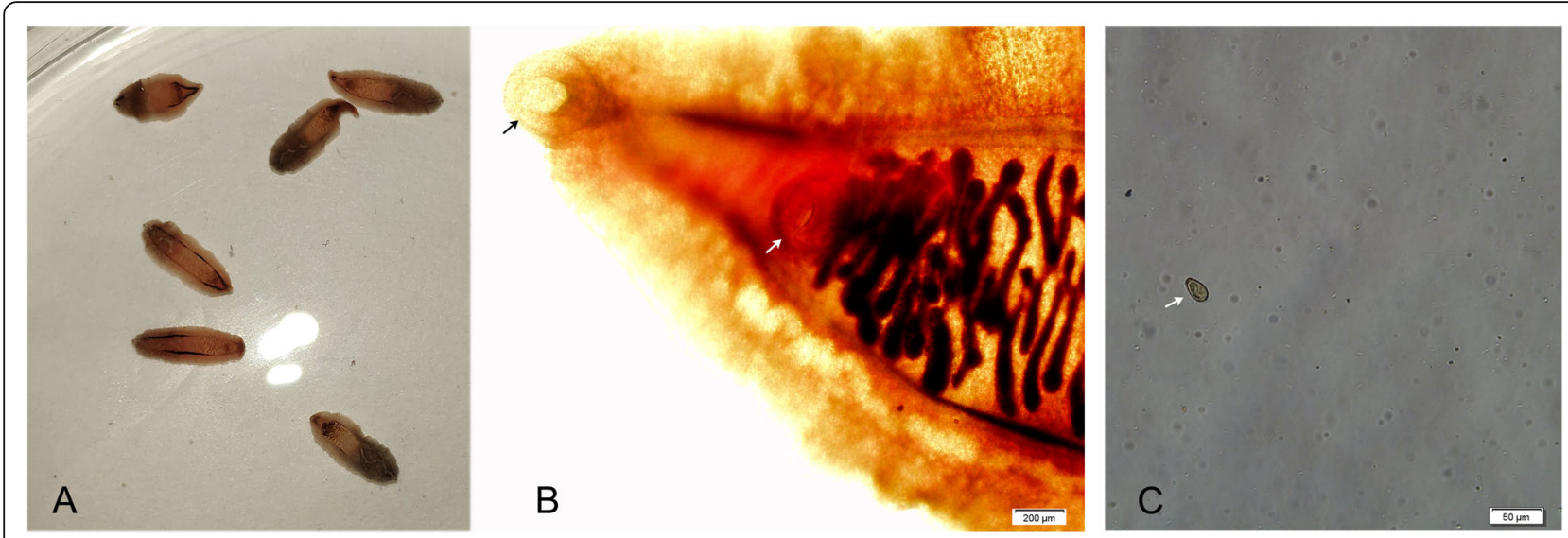

Fig. 2 Morphologic observation of C. sinensis worms and egg. a C. sinensis worms drained from bile. $\mathbf{b}$ The morphologic features of worm with oral sucker (black arrow) and ventral sucker (white arrow). c Egg of C. sinensis from bile

The patient in this study lived in Songyuan city, Jilin province, China, near the Songhua River, which is a heavily endemic area of clonorchiasis [17]. People living around the Songhua River have a deep-rooted custom of eating raw freshwater fish during festivals and feeding this food to cats and dogs, and the prevalence of $C$. sinensis is high. In the meantime, it is common in some endemic regions in China, particularly in the province of Jilin, for the "toilet" to be built close to fish ponds. Eggs of C. sinensis from human excrement are the source of infection for

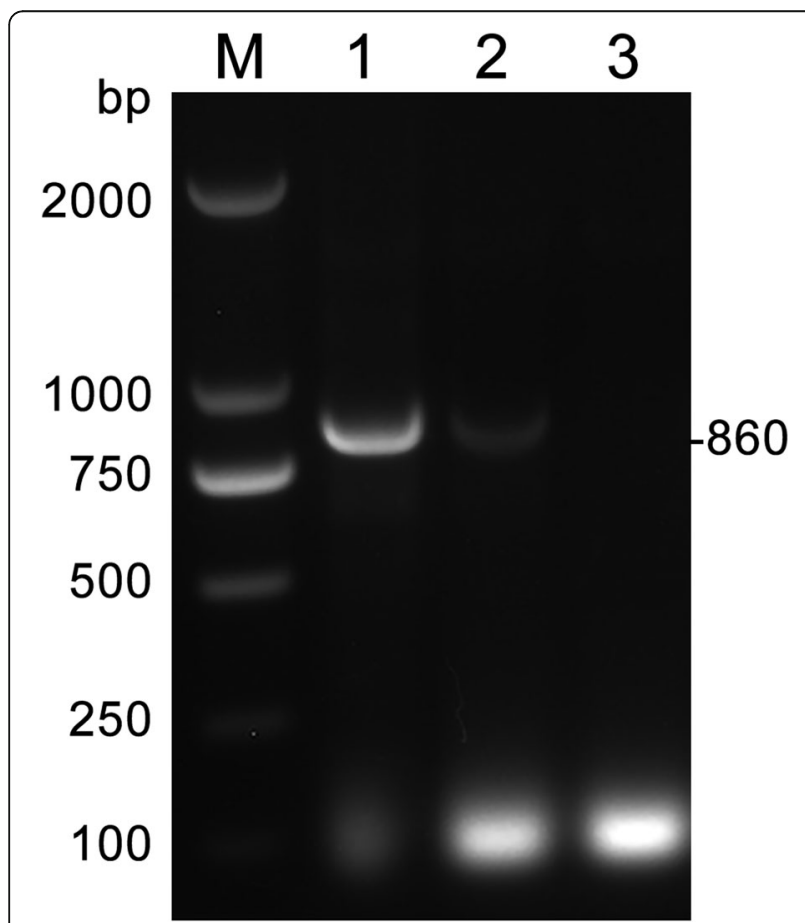

Fig. 3 PCR products on C. sinensis adults and eggs. M, DNA marker; lane 1, C. sinensis adults; lane 2, C. sinensis eggs; lane 3, negative control. The arrow on the right side represents the amplicon of $C$. sinensis intermediate hosts in ponds [1]. The existence of a large number of intermediate and reservoir hosts is closely related to the maintenance and development of the complete life cycle of $C$. sinensis in local areas, leading to long periods of existence of the parasite [1]. Therefore, people around the Songhua River have a high prevalence of C. sinensis infection.

Chronic clonorchiasis in the gallbladder and bile ducts leads to various complications, such as cholecystitis, cholangitis, gallstones, and cholangiocarcinoma [18]. Several pathogenic mechanisms have been proven, such as the direct invasion of larva, injury caused by worms through adsorption or feeding activities on the mucosa of the bile ducts, and biliary obstruction by the worms and stones formed by excretory-secretory products (ESPs) or eggs [19-21]. Once the bile ducts are obstructed, this condition provides a favorable environment for secondary bacterial infection [6]. In addition, ESPs produced by worms increase free radicals, which could promote the production of pro-inflammatory cytokines through the NF- $\mathrm{kB}$ pathway [22]. Patients that presented with acute abdomen, calculous cholecystitis, jaundice and obstructive jaundice caused by $C$. sinensis infection have been reported in recent years $[13,14,23]$. However, acute shock caused by $C$. sinensis infection was not reported. In this rare case, acute cholecystitis caused by $C$. sinensis resulted in shock, which is a rare symptom of clonorchiasis. Not considering the life history of the patient in the epidemic area and neglecting the increase in eosinophil count led to misdiagnosis. Even if cholecystectomy is feasible, conservative antihelmintic treatment should be tried first.

The traditional diagnosis method of clonorchiasis is to detect the characteristic operculated ova in stool. This method is simple, rapid, and inexpensive; however, it is difficult to find eggs during early infection and biliary obstruction [24]. Patients with low-intensity and moderateintensity infection had a higher rate of misdiagnosis, for 
which rich practical experience was needed. In China, it has been reported that the misdiagnosis rate of individuals with clonorchiasis was $57.1 \%$ of 49 patients, $52.5 \%$ of 40 patients, and $84.18 \%$ of 196 patients in different endemic areas [25-27]. Clonorchiasis was mainly misdiagnosed as hepatitis, cholecystitis, and gallstones [27]. Imaging and serological diagnoses are used as auxiliary methods to improve the diagnosis [28]. PCR is a complementary tool used for the diagnosis that can detect $C$. sinensis in patients with low-intensity infection and may help eliminate cross-reactions between the pathogen and other parasite species [29]. Therefore, we used PCR for diagnosis in this case.

In conclusion, shock caused by C. sinensis is rare in the clinical environment. This case improves our awareness of the impacts of clonorchiasis. The misdiagnosis rate of clonorchiasis is high in the clinical environment because the disease has no typical symptoms or is asymptomatic. Moreover, auxiliary methods are difficult to accurately diagnose without detecting eggs in the stool. Therefore, when a patient present with the above mentioned symptoms and imaging shows cholecystitis, cholelithiasis, increased bilirubin and eosinophils in blood, C. sinensis infection should be considered. Most importantly, this detection method will help reduce the misdiagnosis of clonorchiasis and unnecessary cholecystectomy.

\section{Abbreviations \\ CT: Computed Tomography; MRCP: Magnetic Resonance \\ Cholangiopancreatography; PCR: Polymerase Chain Reaction}

\section{Acknowledgements}

We sincerely appreciate that the patient agreed to publish this report.

\section{Authors' contributions}

WN, TB and HYH carried out the literature search and drafted the first version of the manuscript. $L X L, L M Y$ and $W X L$ were responsible for designing. BX, LYX and YY revised the review, LSC, HS, WXY performed the experiments. All authors read and approved the final manuscript.

\section{Funding}

This study was supported by The National Key Research and Development Program of China (2017YFC1601206, 2017YFC1601202, 2018YFC1602504) and Guangdong Innovative and Enterpreneurial Research Team Program (NO. 2014ZT05S123).

\section{Availability of data and materials}

All data generated or analysed during this study are included in this published article.

\section{Ethics approval and consent to participate}

The report of clinical specimen was obtained with written consent and approved by the First Hospital of Jilin University Ethical Committee, and written consent was obtained from patient involved in this study.

\section{Consent for publication}

The patient had signed the Consent form and agreed to publish this report and a copy of written Consent is available for the journal.

\section{Competing interests}

The authors declare that they have no competing interests.

\section{Author details}

'Key Laboratory of Zoonosis Research, Ministry of Education, Institute of Zoonosis/College of Veterinary Medicine, Jilin University, Changchun 130000, China. ${ }^{2}$ Affiliated Zhongshan Hospital of Dalian University, Dalian 116000,

China. Infection Department, the First Hospital of Jilin University, Changchun 130021, China. ${ }^{4}$ Jiangsu Co-innovation Center for Prevention and Control of Important Animal Infectious Diseases and Zoonoses, Yangzhou, China.

Received: 13 May 2019 Accepted: 20 November 2019

Published online: 29 November 2019

\section{References}

1. Lun ZR, Gasser RB, Lai DH, Li AX, Zhu XQ, Yu XB, Fang YY. Clonorchiasis: a key foodborne zoonosis in China. Lancet Infect Dis. 2005;5(1):31-41.

2. Bouvard V, Baan R, Straif K, Grosse Y, Secretan B, El Ghissassi F, BenbrahimTallaa L, Guha N, Freeman C, Galichet L, et al. A review of human carcinogens--part B: biological agents. Lancet Oncol. 2009;10(4):321-2.

3. Furst T, Keiser J, Utzinger J. Global burden of human food-borne trematodiasis: a systematic review and meta-analysis. Lancet Infect Dis. 2012:12(3):210-21.

4. Qian MB, Chen YD, Liang S, Yang GJ, Zhou XN. The global epidemiology of clonorchiasis and its relation with cholangiocarcinoma. Infect Dis Poverty. 2012;1(1):4.

5. Rim HJ. Clonorchiasis: an update. J Helminthol. 2005;79(3):269-81.

6. Qian MB, Utzinger J, Keiser J, Zhou XN. Clonorchiasis. Lancet. 2016; 387(10020):800-10.

7. Marcos LA, Terashima A, Gotuzzo E. Update on hepatobiliary flukes: fascioliasis, opisthorchiasis and clonorchiasis. Curr Opin Infect Dis. 2008;21(5): 523-30.

8. Hong ST, Fang Y. Clonorchis sinensis and clonorchiasis, an update. Parasitol Int. 2012;61(1):17-24

9. Qian MB, Chen YD, Yan F. Time to tackle clonorchiasis in China. Infect Dis Poverty. 2013;2(1):4

10. Kim YH. Pancreatitis in association with Clonorchis sinensis infestation: $C T$ evaluation. AJR Am J Roentgenol. 1999;172(5):1293-6.

11. Choi JH, Kim JH, Kim CH, Jung YK, Yeon JE, Byun KS, Kim I. Pancreatic mucinous cystadenoma of borderline malignancy associated with Clonorchis sinensis. Korean J Internal Med. 2015;30(3):398-401.

12. Li T, He S, Zhao H, Zhao G, Zhu XQ. Major trends in human parasitic diseases in China. Trends Parasitol. 2010;26(5):264-70.

13. Sun Q, Liu X, Hao Y, Li Y, Bai X, Wang F, Liu M. A misdiagnosis of clonorchiasis as gallstone, leading to an unnecessary cholecystectomy: a case report. Am J Emerg Med. 2014;32(11):1442.e1443-5.

14. Hao Y, Bao W, Jin M, Li Y, Wang F. Painless jaundice caused by Clonorchis sinensis infection: a case report. Korean J Parasitol. 2016;54(3):323-7.

15. Attwood HD, Chou ST. The longevity of Clonorchis sinensis. Pathology. 1978; 10(2):153-6.

16. Lim JH. Liver flukes: the malady neglected. Korean J Radiol. 2011;12(3):269-79.

17. Choi MH, Park SK, Li Z, Ji Z, Yu G, Feng Z, Xu L, Cho SY, Rim HJ, Lee SH, et al. Effect of control strategies on prevalence, incidence and re-infection of clonorchiasis in endemic areas of China. PLoS Negl Trop Dis. 2010;4(2): e601.

18. Cho SH, Lee KY, Lee BC, Cho PY, Cheun HI, Hong ST, Sohn WM, Kim TS. Prevalence of clonorchiasis in southern endemic areas of Korea in 2006. Korean J Parasitol. 2008:46(3):133-7.

19. Choi D, Lim JH, Lee KT, Lee JK, Choi SH, Heo JS, Choi DW, Jang KT, Lee NY, Kim S, et al. Gallstones and Clonorchis sinensis infection: a hospital-based case-control study in Korea. J Gastroenterol Hepatol. 2008:23(8 Pt 2):e399-404

20. Zheng S, Zhu Y, Zhao Z, Wu Z, Okanurak K, Lv Z. Liver fluke infection and cholangiocarcinoma: a review. Parasitol Res. 2017;116(1):11-9.

21. Qiao T, Ma RH, Luo ZL, Yang LQ, Luo XB, Zheng PM. Clonorchis sinensis eggs are associated with calcium carbonate gallbladder stones. Acta Trop. 2014; 138:28-37.

22. Nam JH, Moon JH, Kim IK, Lee MR, Hong SJ, Ahn JH, Chung JW, Pak JH. Free radicals enzymatically triggered by Clonorchis sinensis excretorysecretory products cause NF-kappaB-mediated inflammation in human cholangiocarcinoma cells. Int J Parasitol. 2012;42(1):103-13.

23. Liu X, Zhu G, Cai C, Lv Z, Li J. Clonorchiasis sinensis detected by laparoscopic exploration of biliary tracts in two patients with obstructive jaundice. BMC Infect Dis. 2019;19(1):33. 
24. Joo KR, Bang SJ. A bile based study of Clonorchis sinensis infections in patients with biliary tract diseases in Ulsan, Korea. Yonsei Med J. 2005;46(6): 794-8.

25. Zhang CJ, Liu YH. Analysis on misdiagnosis of 28 Clonorchiasis cases. J Norman Bethune Univ Med Sci. 1990;2:188-97 (in chinese).

26. Li L, Duan M, Jiang CD, Li Y. Analysis on clinical, misdiagnosis and missed diagnosis of 40 Clonorchiasis cases. Ji Sheng Chong Bing Yu Gan Ran Xing Ji Bing. 2004;4:187-8 (in chinese).

27. Xie M, Feng JE. Analysis on clinical characteristics and misdiagnosis of 196 Clonorchiasis cases. Zhong Guo Re Dai Yi Xue. 2006;11:2002-4 (in chinese).

28. Choi Bl, Han JK, Hong ST, Lee KH. Clonorchiasis and cholangiocarcinoma: etiologic relationship and imaging diagnosis. Clin Microbiol Rev. 2004;17(3): 540-52 table of contents.

29. Traub RJ, Macaranas J, Mungthin M, Leelayoova S, Cribb T, Murrell KD, Thompson RC. A new PCR-based approach indicates the range of Clonorchis sinensis now extends to Central Thailand. PLoS Negl Trop Dis. 2009;3(1):e367.

\section{Publisher's Note}

Springer Nature remains neutral with regard to jurisdictional claims in published maps and institutional affiliations.

Ready to submit your research? Choose BMC and benefit from:

- fast, convenient online submission

- thorough peer review by experienced researchers in your field

- rapid publication on acceptance

- support for research data, including large and complex data types

- gold Open Access which fosters wider collaboration and increased citations

- maximum visibility for your research: over $100 \mathrm{M}$ website views per year

At BMC, research is always in progress.

Learn more biomedcentral.com/submissions 